\title{
Guaranteed Cost Model Predictive Control-based Driver Assistance System for Vehicle Stabilization Under Tire Parameters Uncertainties
}

\author{
Carlos M. Massera ${ }^{1}$, Marco H. Terra ${ }^{2}$ and Denis F. Wolf ${ }^{1}$
}

\begin{abstract}
Road traffic crashes have been the leading cause of death among young people. Most of these accidents occur when the driver becomes distracted and a loss-of-control situation occurs. Steer-by-Wire systems were recently proposed as an alternative to mitigate such accidents. This technology enables the decoupling of the front wheel steering angles from the driver hand wheel angle and, consequently, the measurement of road/tire friction limits and the development of novel control systems capable of ensuring vehicle stabilization and safety. However, vehicle safety boundaries are highly dependent on tire characteristics which vary significantly with temperature, wear and the tire manufacturing process. Therefore, design of autonomous vehicle and driver assistance controllers cannot assume that these characteristics are constant or known. Thus, this paper proposes a Guaranteed Cost Model Predictive Controller Driver Assistance System able to avoid front and rear tire saturation and to track the drivers intent up to the limits of handling for a vehicle with uncertain tire parameters. Simulation results show the performance of the proposed approach under time-varying uniformly distributed disturbances.
\end{abstract}

\section{INTRODUCTION}

Road traffic crashes are the leading cause of death among young people between 10 and 24 years old [15]. Most of these accidents occur when the driver is unable to maintain the vehicle control due to fatigue or external factors resulting in loss-of-control scenarios [1]. In recent years, both academia and industry have been devoted towards the development of safety systems in order to decrease the number of road accidents.

Steer-by-Wire systems were recently introduced in commercial vehicles [19]. This system eliminates mechanical coupling between the drivers steering wheel and the front road wheels. In 2010, Hsu et al. [10] demonstrated that a Steer-by-Wire system allowed friction estimation based on the steering torque and Hamann et al. [8] proposed a faster converging method based on the Unscented Kalman Filter. The capability to decouple the front wheel steering angles from the driver and to measure saturation limits enable the development of controllers able to predict and avoid vehicle handling saturation limits.

Model Predictive Control (MPC) is a class of optimization-based control algorithms which uses an explicit model of the controlled system to predict its future states [2]. Beal and Gerdes [3] proposed a MPC-based

\footnotetext{
This work was supported by FAPESP grant 2013/24542-7.

${ }^{1}$ Institute of Mathmatics and Computer Science, University of São Paulo, Avenida Trabalhador São-carlense, 400, São Carlos, Brazil massera, denis@icmc.usp.br

${ }^{2}$ São Carlos School of Engineering, University of São Paulo, Avenida Trabalhador São-carlense, 400, São Carlos, Brazil terra@ sc . usp. br
}

Driver Assistance System (DAS) for Steer-by-Wire vehicles able to ensure vehicle handling limits in coordination with a human driver. A linear bicycle model variation, named Affine Force Input (AFI), was also proposed. This model is able to represent the tire dynamics close to a linearization point and to maintain convexity of the optimization problem. Bernardini et al. [4] developed a Hybrid MPC to coordinate steering correction and differential braking. Tire forces were approximated by a piece-wise affine function and the optimization problem of the MPC controller was formulated as a mixed-integer quadratic problem. Massera and Denis [14] previously proposed a MPC-based DAS for Steer-byWire vehicles which ensured vehicle handling limits by incorporating both longitudinal and lateral dynamics by iterative linearizations of a force input nonlinear bicycle model.

Tire characteristics vary significantly with temperature [18], wear [5] and their manufacturing process. Therefore, the design of autonomous vehicles and driver assistance controllers cannot assume that these characteristics are constant or known. The disregard of such uncertainties can lead to poor closed-loop performance of MPCs and, consequently, to the violation of state and control input constraints [17]. Massera et al. [13] have proposed a Robust MPC technique, entitled Guaranteed Cost Model Predictive Control (GCMPC), able to guarantee robust stability, robust feasibility and an upper bound to a MPC optimization problem cost for linear system with multiplicative parametric uncertainties.

This paper proposes a Guaranteed Cost Model Predictive Controller Driver Assistance System able to avoid front and rear tire saturation and to track the drivers intent up to the limits of handling for a vehicle with uncertain tire parameters. The control law is designed such that both the rear and the front tire never saturate and the vehicle never exceeds its maximum stable yaw rate boundaries.

The remainder of the paper is organized as follows: Section II discusses the tire model and its uncertainties; Section III presents the vehicle modeling; Section IV] describes the proposed controller; Section $\mathrm{V}$ reports the experiments performed; and Section $\mathrm{VI}$ provides the final remarks.

\section{TIRE MODEL}

There are three categories of models capable of representing the tire dynamics on saturation situations: Finite element analysis [9] [11]; Empirical data approximation, such as the "Magic Tire Formula" [16]; and the dynamical approximation of the tire by a "brush" model, first proposed by Fiala [6]. The third technique, provides a good compromise 
between its ability to describe tires physical properties and its complexity, since it assumes that tires are always at steady state and tire transients are faster than chassis transients. While a linear model provides a good approximation of tire forces for low slip conditions.

\section{A. Linear Tire Model}

A linear approximation of the lateral tire forces is valid for low slip angle situations and enables the development of a linear model of the vehicle dynamics. For a given tire $i \in\{f, r\}$, the tire lateral force is described by

$$
F_{y i}=-C_{i} \alpha_{i}
$$

where $\alpha_{i}$ is the tire slip angle and $C_{i}$ is the cornering stiffness.

\section{B. Fiala Tire Model}

The Fiala Tire model is parameterized by its slip angle $\left(\alpha_{i}\right)$, cornering stiffness $\left(C_{i}\right)$, static friction coefficient $(\mu)$, tire normal force $\left(F_{z i}\right)$, and the ratio between dynamical and static friction coefficients $\left(R_{\mu, i}\right)$ for a given tire $i \in\{f, r\}$. The lateral tire force for this model is described by

$$
\begin{aligned}
F_{y i}=-f\left(\alpha_{i}\right)+\frac{2-R_{\mu, i}}{3 \mu F_{z i}}\left|f\left(\alpha_{i}\right)\right| & f\left(\alpha_{i}\right)- \\
& -\frac{1-\frac{2}{3} R_{\mu, i}}{\left(3 \mu F_{z i}\right)^{2}} f\left(\alpha_{i}\right)^{3}
\end{aligned}
$$

for the unsaturated case $\left(\left|\alpha_{i}\right| \leq \tan ^{-1}\left(3 \mu F_{z i} / C_{i}\right)\right)$ and

$$
F_{y i}=-\operatorname{sign}\left(\alpha_{i}\right) \mu R_{\mu, i} F_{z i}
$$

for the saturated case $\left(\left|\alpha_{i}\right|>\tan ^{-1}\left(3 \mu F_{z i} / C_{i}\right)\right)$ where $f\left(\alpha_{i}\right)=C_{i} \tan \left(\alpha_{i}\right)$. From (2), the peak lateral force and its slip angle are

$$
\begin{aligned}
q & =\left(1-\frac{2}{3} R_{m u}\right)^{-1}, \\
F_{y i}^{\text {peak }} & =\mu F_{z i}\left(-q+\frac{2-R_{m u}}{3} q^{2}+\frac{1-\frac{2}{3} R_{m u}}{9} q^{3}\right), \\
\alpha_{i}^{\text {peak }} & =\tan ^{-1}\left(\frac{q \mu F_{z i}}{C_{i}}\right) .
\end{aligned}
$$

\section{Parameter Sensitivity}

The normal load $F_{z i}$ does not vary significantly and it is always coupled with the friction coefficient $\mu$. Thus, this study will focus on the sensitivity of $C_{i}, R_{\mu, i}$ and $\mu$.

Let $p_{i}=\left[C_{i}, R_{\mu, i}, \mu\right]^{T}$ be the parameter vector of the $i$-th tire and $p_{i}^{n}$ be the nominal or estimated parameters. Then, the set of possible parametric tire disturbances

$$
\begin{aligned}
\mathcal{W}_{i}=\left\{p \mid p \in \mathbb{R}^{3}, C_{i} \in[0.7,1.3] C_{i}^{a},\right. \\
\left.R_{\mu, i} \in[0.9,1.1] R_{\mu, i}^{a}, \mu \in[0.9,1.1] \mu^{a}\right\}
\end{aligned}
$$

represents uncertainties of $30 \%$ on $C_{i}, 10 \%$ on $R_{\mu, i}$ and $10 \%$ on $\mu$. Figure 1 shows the lateral force profile for each vertex of $\mathcal{W}_{i}$, and also presents the upper bound, the lower bound and the intermediary force profiles.

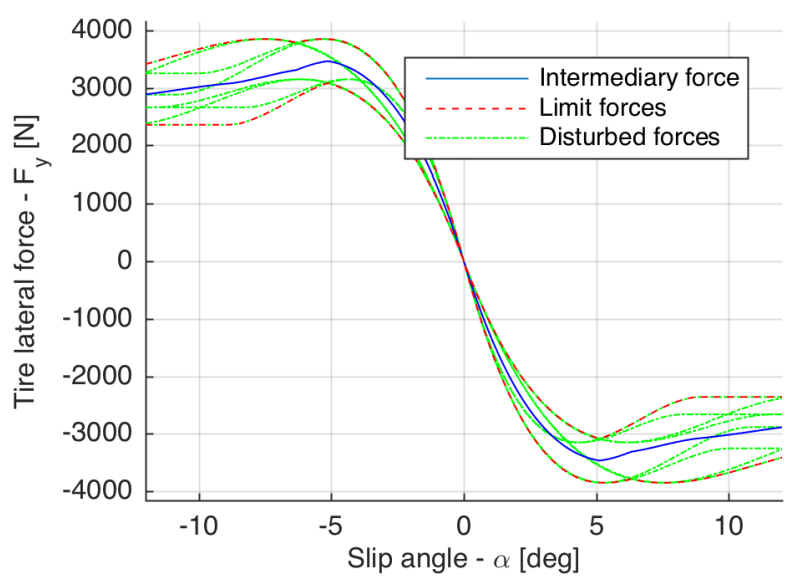

Fig. 1: Fiala tire model lateral force subject to parametric disturbances of $30 \%$ on $C_{i}, 10 \%$ on $R_{\mu, i}$ and $10 \%$ on $\mu$.

Let $F_{y i}^{\text {sup }}\left(\alpha_{i}\right)=\sup _{p_{i} \in \mathcal{W}_{i}} F_{y i}\left(\alpha_{i}\right)$ and $F_{y i}^{\text {inf }}\left(\alpha_{i}\right)=$ $\inf _{p_{i} \in \mathcal{W}_{i}} F_{y i}\left(\alpha_{i}\right)$ be the upper and lower bound forces for a given slip angle, respectively. Then, the intermediary force $\bar{F}_{y i}\left(\alpha_{i}\right)$ and the force deviation are defined by

$$
\begin{aligned}
\bar{F}_{y i}\left(\alpha_{i}\right) & =\left(F_{y i}^{\text {sup }}\left(\alpha_{i}\right)+F_{y i}^{\text {inf }}\left(\alpha_{i}\right)\right) / 2 \\
\partial F_{y i}\left(\alpha_{i}\right) & =\left(F_{y i}^{\text {sup }}\left(\alpha_{i}\right)-F_{y i}^{\text {inf }}\left(\alpha_{i}\right)\right) / 2
\end{aligned}
$$

From we conclude that

$$
\begin{aligned}
& \forall p_{i} \in \mathcal{W}_{i} \exists \gamma_{f} \in \mathbb{R},\left|\gamma_{f}\right| \leq 1: \\
& \quad F_{y i}\left(\alpha_{i}\right)=\bar{F}_{y i}\left(\alpha_{i}\right)+\gamma_{f} \partial F_{y i}\left(\alpha_{i}\right) .
\end{aligned}
$$

Considering definitions similar to 9 for the local cornering stiffness $\widehat{C}_{i}\left(\alpha_{i}\right)=-\partial F_{y i}\left(\alpha_{i}\right) / \partial \alpha_{i}$ results

$$
\begin{aligned}
\forall p_{i} \in \mathcal{W}_{i} \exists \gamma_{c} \in \mathbb{R}, & \left|\gamma_{c}\right| \leq 1: \\
& \widehat{C}_{i}\left(\alpha_{i}\right)=\bar{C}_{i}\left(\alpha_{i}\right)+\gamma_{c} \partial C_{i}\left(\alpha_{i}\right) .
\end{aligned}
$$

\section{VEHICLE MODEL}

This section describes the linear bicycle model, the AFI bicycle model, proposed by Beal and Gerdes [3], and the proposed parametric uncertain AFI bicycle model. The bicycle model is a simplification of the vehicle dynamics where the left and right front wheels are replaced by a virtual front wheel in the center of the front axle and, analogously, the left and right rear wheels are replaced by a virtual wheel in the center of the rear axle. A representation of a vehicle on this form is shown in Figure 2

\section{A. Linear Bicycle Model}

The linear dynamical model is a two-state model, which describes both yaw-rate $r$ and lateral speed $v_{y}$ of a vehicle based on the bicycle model [5]. It uses low angle and constant longitudinal speed assumptions $(\cos (x) \approx 1, \sin (x) \approx x$, $\tan (x) \approx x$ and $\dot{v}_{x} \approx 0$ ) [14]. Its equations of motion are given by

$$
\dot{v}_{y}=\frac{F_{y f}+F y r}{m}-v_{x} r, \dot{r}=\frac{a F_{y f}-b F_{y r}}{I_{z}}
$$




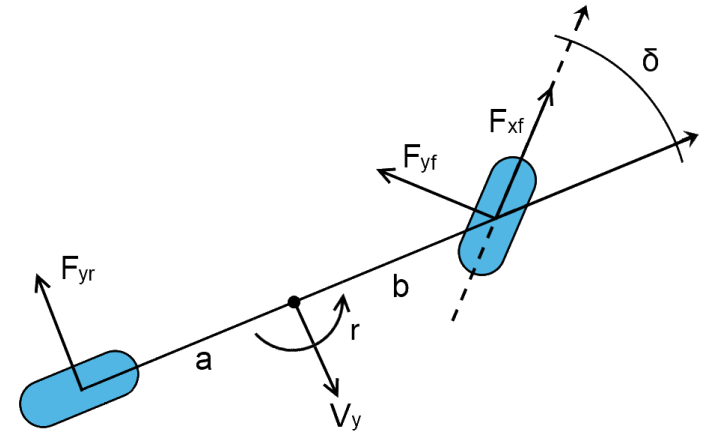

Fig. 2: Bicycle model representation

in which $a, b, m$ and $I_{z}$ are the distance from the front axle to the center of gravity, the distance from the rear axle to the center of gravity, the vehicle mass and the vehicle yaw moment of inertia, respectively. The tire forces are described by the linear tire model from (1) with approximate slip angles

$$
\alpha_{f} \approx \frac{v_{y}+a r}{v_{x}}-\delta, \alpha_{r} \approx \frac{v_{y}-b r}{v_{x}}
$$

in which delta is the steering angle. Therefore, the linear bicycle system can be represented in state-space form $\dot{x}=$ $A_{l} x+B_{l} u$ with $x=\left[v_{y}, r\right]^{T}, u=\delta$ and

$$
A_{l}=\left[\begin{array}{cc}
-\frac{C_{f}+C_{r}}{m v_{x}} & -\frac{a C_{f}-b C_{r}}{m v_{x}}-v_{x} \\
-\frac{a C_{f}-b C_{r}}{I_{z} v_{x}} & -\frac{a^{2} C_{f}+b^{2} C_{r}}{I_{z} v_{x}}
\end{array}\right], B_{l}=\left[\begin{array}{c}
\frac{C_{f}}{m} \\
\frac{a C_{f}}{I_{z}}
\end{array}\right]
$$

\section{B. Affine Force Input Bicycle Model}

The AFI bicycle model was first proposed by Beal and Gerdes [3]. The front tire forces are abstracted from the system dynamics, such that the system input is chosen to be $F_{y f}$, instead of $\delta$. Also, the rear tire forces are approximated by a first order Taylor series

$$
F_{y r}\left(\alpha_{r}\right) \approx F_{y r}\left(\widehat{\alpha}_{r}\right)-\left(\alpha_{r}-\widehat{\alpha}_{r}\right) \widehat{C}_{r}\left(\widehat{\alpha}_{r}\right) .
$$

Thus, the AFI bicycle system can be represented in the affine state-space form $\dot{x}=A_{a f i} x+B_{a f i} u+c_{a f i}$ with $x=$ $\left[v_{y}, r\right]^{T}, u=F_{y f}$ and

$$
\begin{gathered}
A_{a f i}=\left[\begin{array}{cc}
-\frac{\widehat{C}_{r}\left(\widehat{\alpha}_{r}\right)}{m v_{x}} & \frac{b \widehat{C}_{r}\left(\widehat{\alpha}_{r}\right)}{m v_{x}}-v_{x} \\
-\frac{b \widehat{C}_{r}\left(\widehat{\alpha}_{r}\right)}{I_{z} v_{x}} & -\frac{b^{2} \widehat{C}_{r}\left(\widehat{\alpha}_{r}\right)}{I_{z} v_{x}}
\end{array}\right], \\
B_{a f i}=\left[\begin{array}{c}
\frac{1}{m} \\
\frac{a}{I_{z}}
\end{array}\right], c_{a f i}=\left[\begin{array}{c}
\frac{\widehat{F}_{y r}\left(\widehat{\alpha}_{r}\right)+\widehat{C}_{r}\left(\widehat{\alpha}_{r}\right) \widehat{\alpha}_{r}}{m} \\
-b \frac{\widehat{F}_{y r}\left(\widehat{\alpha}_{r}\right)+\widehat{C}_{r}\left(\widehat{\alpha}_{r}\right) \widehat{\alpha}_{r}}{I_{z}}
\end{array}\right]
\end{gathered}
$$

This model is able to represent the vehicle lateral dynamics up to the limits of handling. However, it is only valid for rear slip angles close to the linearization point $\hat{\alpha_{r}}$.

\section{Parametric Uncertain AFI Bicycle Model}

Disturbance on tire parameters has direct influence on the system dynamics of the AFI model. Front tire disturbances causes a multiplicative uncertainty on the input $u$, while rear tire disturbances results in multiplicative uncertainties on matrices $A$ and $c$.
The substitution of the parametrically uncertain tire model from $(10)$ and $(11)$ on the AFI system dynamics from (16) yields

$$
\begin{gathered}
A_{a f i}=\left[\begin{array}{cc}
-\frac{\bar{C}_{i}\left(\widehat{\alpha}_{r}\right)+\gamma_{c} \partial C_{r}\left(\widehat{\alpha}_{r}\right)}{m v_{x}} & \frac{b\left[\bar{C}_{r}\left(\widehat{\alpha}_{r}\right)+\gamma_{c} \partial C_{r}\left(\widehat{\alpha}_{r}\right)\right]}{m v_{x}}-v_{x} \\
-\frac{b\left[\bar{C}_{r}\left(\widehat{\alpha}_{r}\right)+\gamma_{c} \partial C_{r}\left(\widehat{\alpha}_{r}\right)\right]}{I_{z} v_{x}} & -\frac{b^{2}\left[\bar{C}_{r}\left(\widehat{\alpha}_{r}\right)+\gamma_{c} \partial C_{r}\left(\widehat{\alpha}_{r}\right)\right]}{I_{z} v_{x}}
\end{array}\right] \\
B_{a f i}=\left(1+\gamma_{b} \frac{\partial F_{y f}\left(\widehat{\alpha}_{f}\right)}{\bar{F}_{y f}\left(\widehat{\alpha}_{f}\right)}\right)\left[\begin{array}{c}
\left.\frac{1}{m}\right] \\
\frac{a}{I_{z}}
\end{array}\right] \\
c_{a f i}=\left[\begin{array}{c}
\frac{\left.\bar{F}_{y r}\left(\widehat{\alpha}_{r}\right)\right)+\gamma_{f} \partial F_{y r}\left(\widehat{\alpha}_{r}\right)+\left[\bar{C}_{r}\left(\widehat{\alpha}_{r}\right)+\gamma_{c} \partial C_{r}\left(\widehat{\alpha}_{r}\right)\right] \widehat{\alpha}_{r}}{m} \\
-b \frac{\left.\bar{F}_{y r}\left(\widehat{\alpha}_{r}\right)\right)+\gamma_{f} \partial F_{y r}\left(\widehat{\alpha}_{r}\right)+\left[\bar{C}_{r}\left(\widehat{\alpha}_{r}\right)+\gamma_{c} \partial C_{r}\left(\widehat{\alpha}_{r}\right)\right] \widehat{\alpha}_{r}}{I_{z}} .
\end{array}\right]
\end{gathered}
$$

This system dynamics can be rewritten in the parametric uncertain affine state-space form

$$
\begin{array}{r}
\dot{x}=\left(\bar{A}_{a f i}+H_{a f i} \Delta_{a f i} E_{a, a f i}\right) x+\left(\bar{B}_{a f i}+H_{a f i} \Delta E_{b, a f i}\right) u+ \\
+\left(\bar{c}_{a f i}+H_{a f i} \Delta_{a f i} E_{c, a f i}\right)
\end{array}
$$

with

$$
\begin{aligned}
& \bar{A}_{a f i}=\left[\begin{array}{cc}
-\frac{\bar{C}_{i}\left(\widehat{\alpha}_{r}\right)}{m v_{x}} & \frac{b \bar{C}_{i}\left(\widehat{\alpha}_{r}\right)}{m v_{x}}-v_{x} \\
-\frac{b \bar{C}_{i}\left(\widehat{\alpha}_{r}\right)}{I_{z} v_{x}} & -\frac{b^{2} \bar{C}_{i}\left(\widehat{\alpha}_{r}\right)}{I_{z} v_{x}}
\end{array}\right] \\
& \bar{B}_{a f i}=\left[\begin{array}{c}
\frac{1}{m} \\
\frac{a}{I_{z}}
\end{array}\right] \\
& \bar{c}_{a f i}=\left[\begin{array}{c}
\frac{\bar{F}_{y r}\left(\widehat{\alpha}_{r}\right)+\bar{C}_{r}\left(\widehat{\alpha}_{r}\right) \widehat{\alpha}_{r}}{m} \\
-b \frac{\bar{F}_{y r}\left(\widehat{\alpha}_{r}\right)+\bar{C}_{r}\left(\widehat{\alpha}_{r}\right) \widehat{\alpha}_{r}}{I_{z}}
\end{array}\right] \\
& H_{a f i}=\left[\begin{array}{cc}
m^{-1} & m^{-1} \\
-b I_{z}^{-1} & a I_{z}^{-1}
\end{array}\right] \\
& E_{a, a f i}=\left[\begin{array}{cc}
-v_{x}^{-1} \partial C_{r}\left(\widehat{\alpha}_{r}\right) & b v_{x}^{-1} \partial C_{r}\left(\widehat{\alpha}_{r}\right) \\
0 & 0 \\
0 & 0
\end{array}\right] \\
& E_{b, a f i}=\left[\begin{array}{lll}
0 & 0 & \partial F_{y f}
\end{array}\right]^{T} \\
& E_{c, a f i}=\left[\begin{array}{lll}
\partial C_{r}\left(\widehat{\alpha}_{r}\right) \widehat{\alpha}_{r} & \partial F_{y r}\left(\widehat{\alpha}_{r}\right) & 0
\end{array}\right]^{T} \\
& \Delta_{a f i}=\left[\begin{array}{ccc}
\gamma_{f} & \gamma_{c} & 0 \\
0 & 0 & \gamma_{g}
\end{array}\right]
\end{aligned}
$$

\section{CONTROLLER DESIGN}

The controllers should primarily ensure driver and vehicle safety, avoiding unsafe yaw rates and tire saturation. Secondarily, it should follow the drivers intent as close as possible and avoid uncomfortable and unnecessary interventions.

\section{A. System and Functional Modeling}

Since the majority of drivers guide their vehicles on state regions where the linear model is valid, the linear bicycle model was chosen to represent the drivers intent for the vehicle. Whereas the parametric uncertain affine force input model was chosen to depict the vehicle dynamics, since it correctly models the vehicle dynamics up to the limits of handling and account for tire parameter uncertainties.

The system state was defined as $x=$ $\left[v_{y}^{r e f}, r^{r e f}, \delta^{r e f}, v_{y}, r, 1\right]^{T} \quad$ where $v_{y}^{r e f}$ and $r^{r e f}$ are the reference lateral velocity and yaw rate, respectively, $\delta^{r e f}$ 
is the drivers hand wheel angle, $v_{y}$ and $r$ are the effective vehicle lateral velocity and yaw rate, respectively, and there is an one-valued state to incorporate the affine terms of the AFI model. The control input was defined as $u=F_{y f}$. Therefore, the continuous time system dynamics can be described as $\dot{x}=\left(A+H_{c} \Delta E_{a}\right) x+\left(B+H_{c} \Delta E_{b}\right) u$ with

$$
A=\left[\begin{array}{cccc}
A_{l} & B_{l} & & \mathbb{O}_{2,3} \\
\mathbb{O}_{1,2} & 1 & \mathbb{O}_{1,3} \\
\mathbb{O}_{3,3} & \bar{A}_{a f i} & \bar{c}_{a f i} \\
& \mathrm{O}_{1,5} & & 1
\end{array}\right], B=\left[\begin{array}{c}
0 \\
0 \\
\bar{B}_{a f i} \\
0
\end{array}\right],
$$

$H_{c}=\left[\mathrm{O}_{2,3}, H_{a f i}^{T}, \mathbb{O}_{2,1}\right]^{T}, \quad \Delta=\Delta_{a f i}, \quad E_{a}=$ $\left[\mathrm{O}_{3,3}, E_{a, a f i}, E_{c, a f i}\right]$ and $E_{b}=E_{b, a f i}$ in which $\mathbb{O}_{i, j}$ is a zero-valued matrix with $i$ rows and $j$ columns.

This system was discretized with a first-order hold on $w=$ $\Delta E_{a} x+\Delta E_{b} u$ such that

$$
\left[\begin{array}{ccc}
F & G & H \\
\mathbb{O}_{3,6} & \mathbb{I}_{3,3}
\end{array}\right]=\exp \left(T_{s}\left[\begin{array}{ccc}
A & B & H_{c} \\
& \mathbb{O}_{3,9}
\end{array}\right]\right)
$$

in which $T_{s}$ is the sampling time in seconds and $\mathbb{I}_{i, j}$ is an identity matrix with $i$ rows and $j$ columns.

The controller objective was designed as the quadratic minimization of $v_{y}^{r e f}-v_{y}, r^{r e f}-r$ and $F_{y f}$ in discrete time. Thus, it was represented in the form

$$
J\left(x_{0}, \mathbf{u}, N\right)=x_{N}^{T} S x_{N}+\sum_{k=0}^{N} x_{k}^{T} Q x+u_{k}^{T} R u_{k}
$$

where $S$ is the terminal cost,

$$
Q=\left[\begin{array}{cccccc}
W_{v_{y}} & 0 & 0 & -W_{v_{y}} & 0 & 0 \\
0 & W_{r} & 0 & 0 & -W_{r} & 0 \\
0 & 0 & 0 & 0 & 0 & 0 \\
-W_{v_{y}} & 0 & 0 & W_{v_{y}} & 0 & 0 \\
0 & -W_{r} & 0 & 0 & W_{r} & 0 \\
0 & 0 & 0 & 0 & 0 & 0
\end{array}\right]
$$

and $R=W_{F_{y f}}$ with $W_{v_{y}}, W_{r}, W_{F_{y f}}>0$ such that $Q \succeq 0$ and $R \succ 0$.

\section{B. Safety Handling Constraints}

Vehicle loss-of-control situations occur when one or more tires saturate, therefore maintaining vehicle controllability is analogous to avoiding tire saturation.

Beal and Gerdes [3] proposed a Envelope Controller where a constraints to limit the rear slip angle and another to limit the yaw rate are used for rear wheel saturation avoidance. From the slip angle definition, the rear slip limit is

$$
\left|\tan ^{-1}\left(\frac{v_{y}-b r}{v_{x}}\right)\right| \leq \alpha_{r}^{\max }
$$

in which $\alpha^{\text {max }}=\alpha^{\text {peak }}$ to avoid skidding scenarios. Therefore, the rear slip constraint is represented by

$$
-v_{x} \tan \left(\alpha_{r}^{\text {peak }}\right) \leq v_{y}-b r \leq v_{x} \tan \left(\alpha_{r}^{\text {peak }}\right) .
$$

The yaw rate constraint ensures the invariability of the operation envelope. It guarantees that the yaw rate value can be maintained at steady-state. Thus, the maximum yaw rate is [14]

$$
r^{\max }=\frac{\mu}{v_{x}} \frac{a b+\max (a, b)^{2}}{\min (a, b)(a+b)}
$$

and the yaw rate constraint is

$$
-r^{\max } \leq r \leq r^{\max } .
$$

The force limits for the front tire are given by

$$
F_{x f}^{2}+F_{y f}^{2} \leq \mu^{2} F_{z f}^{2} .
$$

Since lateral forces have higher influence on vehicle safety the longitudinal force is assumed to be chosen such that

$$
\left|F_{x f}\right| \leq \sqrt{\mu^{2} F_{z f}^{2}-F_{y f}^{2}} .
$$

Therefore, the front tire lateral force is constrained by

$$
-\mu F_{z f} \leq F_{y f} \leq \mu F_{z f}
$$

and the longitudinal forces will use any remainder available force.

All constraints presented are represented by their generic form $M x_{k}+N u_{k} \leq o$ where

$$
M=\left[\begin{array}{cccccc}
0 & 0 & 0 & 1 & -b & 0 \\
0 & 0 & 0 & -1 & b & 0 \\
0 & 0 & 0 & 0 & 1 & 0 \\
0 & 0 & 0 & 0 & -1 & 0 \\
0 & 0 & 0 & 0 & 0 & 0 \\
0 & 0 & 0 & 0 & 0 & 0
\end{array}\right], N=\left[\begin{array}{c}
0 \\
0 \\
0 \\
0 \\
1 \\
-1
\end{array}\right]
$$

and

$$
o=\left[\begin{array}{cccccc}
1 & 1 & 0 & 0 & 0 & 0 \\
0 & 0 & 1 & 1 & 0 & 0 \\
0 & 0 & 0 & 0 & 1 & 1
\end{array}\right]^{T}\left[\begin{array}{c}
v_{x} \tan \left(\alpha_{r}^{\text {peak }}\right) \\
r^{m a x} \\
\mu F_{z f}
\end{array}\right] .
$$

\section{Guaranteed Cost Model Predictive Controller}

The GCMPC is defined by the optimization problem

$$
\begin{aligned}
J^{*}\left(x_{0}, N\right)<\inf _{\mathbf{v}} & \sum_{k=i}^{N-1} v_{k} \bar{R}_{k} v_{k}+x_{i}^{T} S_{0} x_{i} \\
\text { s.t. } & x_{k+1}=\left(F-G K_{k}\right) x_{k}+G v_{k} \\
& \left(M^{(i)}-N^{(i)} K_{k}\right) x_{k}+N^{(i)} v_{k}+ \\
& +\bar{\Phi}_{k, i}(\mathbf{x}, \mathbf{v}) \leq o^{(i)}
\end{aligned}
$$

where $M^{(i)}, N^{(i)}, o^{(i)}$ are the $i$-th row of matrices $M, N$ and vector $o$, respectively, $S_{0}$ is the guaranteed cost matrix, $K_{k}$ is the guaranteed cost control at timestep $k, u_{k}=-K_{k} x_{k}+v_{k}$, $\bar{\Phi}_{k, i}(\mathbf{x}, \mathbf{v})$ is the robustness margin, defined by

$$
\begin{aligned}
\bar{\Phi}_{k, i}(\mathbf{x}, \mathbf{v}) & =\sum_{j=0}^{k-1}\left\|\widetilde{A}_{k}^{(i)} \widetilde{F}^{k-j-1} H\right\|_{1} \bar{\phi}_{j, 1}(\mathbf{x}, \mathbf{v}), \\
\bar{\phi}_{k}(\mathbf{x}, \mathbf{v}) & =\phi_{k}\left(x_{k}, v_{k}\right)+\sum_{i=0}^{k-1} c(k, i) \phi_{i}\left(x_{i}, v_{i}\right), \\
c(k, i) & =\rho_{k-i-1}+\sum_{j=0}^{k-i-2} \rho_{j} c(k-j-1, i),
\end{aligned}
$$


(a)

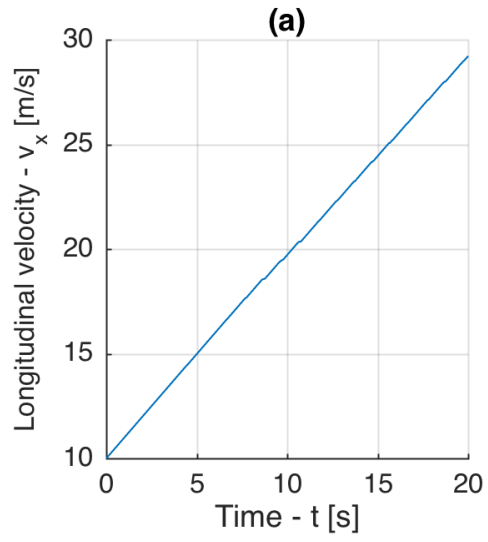

(d)

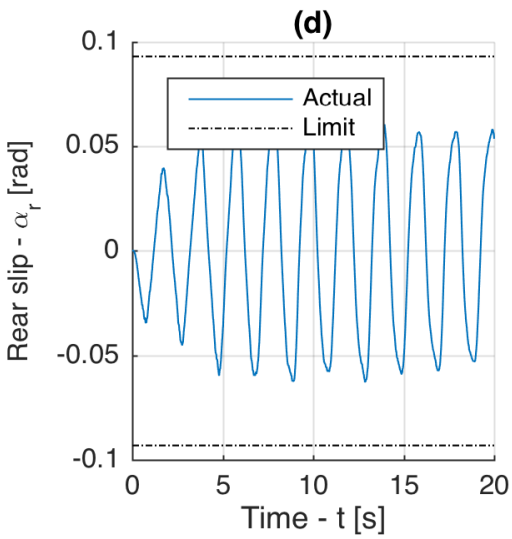

(b)

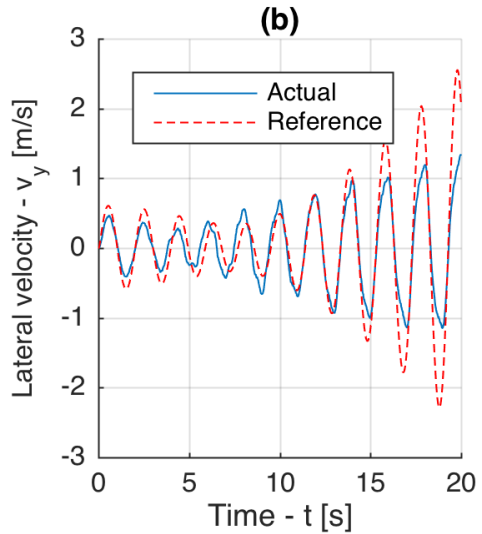

(e)

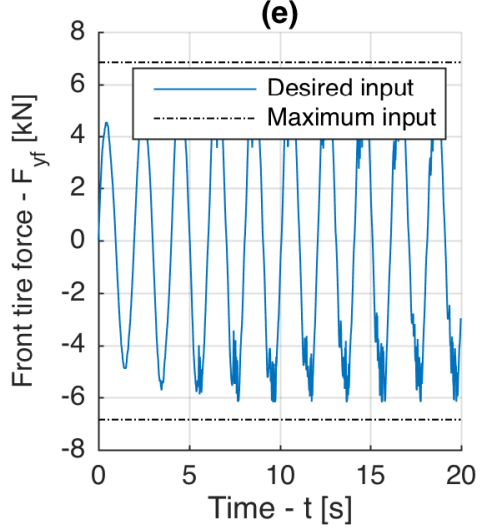

(c)

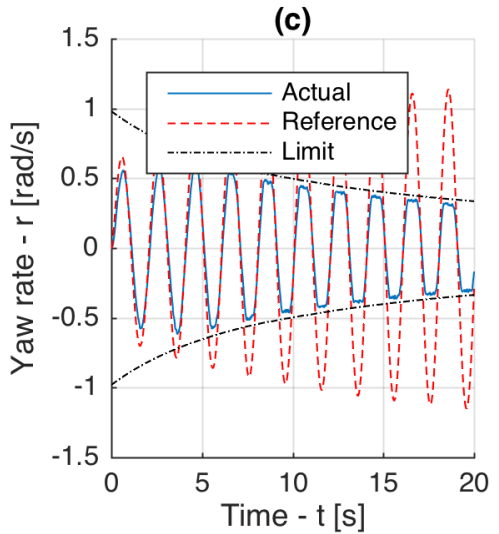

(f)

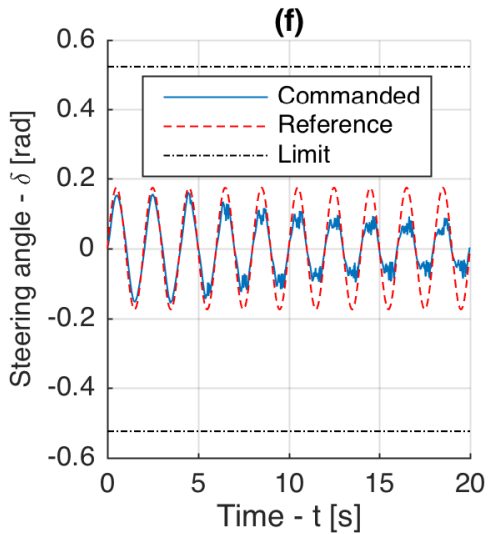

Fig. 3: Simulation results for a slalom maneuver. (a), (b) and (c) present the longitudinal velocity, lateral velocity and yaw rate, respectively. (d), (e) and (f) show the rear slip angle, the desired front tire force and the commanded steering angle, respectively.

where $\phi_{k}(x, v)=\left\|\widetilde{E}_{1, k} x+E_{2} v\right\|_{2}$ is the disturbance boundary, $\rho_{i}=\left\|E_{1} \widetilde{F}^{i} H\right\|_{2}$ is the disturbance propagation norm, $\mathbf{x}=\left\{x_{k} \mid k \in[0, N]\right\}$ is the sequence of states, $\mathbf{u}=\left\{u_{k} \mid k \in[0, N-1]\right\}$ is the sequence of inputs, $\mathbf{v}=\left\{v_{k} \mid k \in[0, N-1]\right\}$ is the sequence of feasibility offsets, $\bar{R}_{k}=R+\epsilon^{-1} E_{b}^{T} E_{b}+G^{T} X_{k+1} G$ is the offset cost matrix, $X_{k}=\left(S^{-1}+\epsilon H H^{T}\right)^{-1}, \epsilon>0$ is obtained such that $\operatorname{tr}(S)$ is bounded and minimal and $J^{*}\left(x_{0}, N\right)$ is the cost of the parametric uncertain MPC optimization problem

$$
\begin{array}{rl}
J^{*}\left(x_{0}, N\right)=\inf _{\mathbf{u}} & J\left(x_{0}, \mathbf{u}, N\right) \\
\text { s.t. } & x_{k+1}=F x_{k}+G u_{k} \\
& M x_{k}+N u_{k} \leq o
\end{array}
$$

For further details on the method and its robustness proofs, the reader should refer to [13] and to the numerical example implementation available online ${ }^{1}$.

\section{Driver Assistance Controller Implementation}

Since the system dynamics depend on $v_{x}$ and $\hat{\alpha}_{r}$, gain scheduling was used to obtain matrices $F, G, E_{a}, S_{0}, X$,

${ }^{1}$ The numerical example source code for the GCMPC is available at: https://github.com/cmasseraf/gcmpc
$K_{k}$ and $\widetilde{K}$. These values were then used in conjunction with the current state $x_{0}$ as inputs to a multi-parametric Quadratic Program (mpQP) designed using YALMIP [12] and Gurobi [7] to represent the optimization problem from (40). The desired front tire force was obtained as $F_{y f}=-K_{0} x_{0}+v_{0}$ and the steering angle was obtained based on the inverse intermediary front tire lateral force $\left(\alpha_{f}=\bar{F}_{y f}^{-1}\left(F_{y f}\right)\right)$

$$
\delta=\tan ^{-1}\left(\frac{v_{y}+a r}{v_{x}}\right)-\bar{F}_{y f}^{-1}\left(F_{y f}\right) .
$$

Since it is not desired to saturate the front tire, this inverse function considers only the region where the intermediary tire force is monotonic $\left(\alpha_{f} \in\left[-\alpha_{f}^{\text {peak }}, \alpha_{f}^{\text {peak }}\right]\right)$.

Table I presents the parameters values used for the proposed controller.

\section{SIMULATION RESULTS}

The simulation scenario used for validation consists of a vehicle at $10 \mathrm{~m} / \mathrm{s}$ accelerating at $1 \mathrm{~m} / \mathrm{s}^{2}$ for $20 \mathrm{~s}$ on a slalom maneuver, where the hand wheel angle is a sine with

\footnotetext{
${ }^{2}$ Parameter dynamically evaluated during control loop
} 
TABLE I: Controller Parameters

\begin{tabular}{|lccc|}
\hline Parameter & Symbol & Value & Unit \\
\hline Prediction horizon & $N$ & 15 & - \\
Lateral Speed weight & $W_{v_{y}}$ & 1 & $\mathrm{~s}^{2} / \mathrm{m}^{2}$ \\
Yaw rate weight & $W_{r}$ & $10^{6}$ & $\mathrm{~s}^{2} / \mathrm{rad}^{2}$ \\
Lateral force weight & $W_{F_{y f}}$ & $10^{-10}$ & $1 / \mathrm{N}^{2}$ \\
Maximum yaw rate & $r^{\text {max }}$ & 2 & $\mathrm{rad} / \mathrm{s}$ \\
Maximum rear slip angle & $\alpha_{r}^{\text {peak }}$ & 2 & $\mathrm{rad}$ \\
Sampling Time & $T_{s}$ & 0.02 & $\mathrm{~s}$ \\
\hline
\end{tabular}

$0.5 \mathrm{~Hz}$ frequency and $10 \mathrm{deg}$ amplitude. Table $\mathrm{I}$ presents the simulated vehicle parameters.

TABLE II: Simulated vehicle parameters

\begin{tabular}{|lccc|}
\hline Parameter & Symbol & Value & Unit \\
\hline Vehicle mass & $m$ & 1231 & $\mathrm{Kg}$ \\
Inertia Moment $z$ & $I_{z}$ & 2034.5 & $\frac{\mathrm{Kg}}{\mathrm{m}^{2}}$ \\
Front axle distance to CG & $a$ & 1.07 & $\mathrm{~m}$ \\
Back axle distance to CG & $b$ & 1.40 & $\mathrm{~m}$ \\
Front tire cornering stiffness & $C_{f}$ & 100000 & $\frac{\mathrm{N}}{\mathrm{rad}}$ \\
Rear tire cornering stiffness & $C_{r}$ & 130000 & $\frac{\mathrm{N}}{\mathrm{rad}}$ \\
Tire-road friction coefficient & $\mu$ & 1 & - \\
Friction coefficient ratio & $R_{\mu, i}$ & 0.8 & - \\
Cornering stiffness uncertainty & - & 20 & $\%$ \\
Tire-road friction uncertainty & - & 10 & $\%$ \\
Friction ratio uncertainty & - & 10 & $\%$ \\
\hline
\end{tabular}

Simulation results are shown in Figure 3, where the tire parameters were chosen to be time-varying and uniformly distributed within the predetermined boundaries. The initial state of the evaluated scenario was $x=[0,0,0,0,0,1]$ and $v_{x}=10$.

Since the vehicle has natural under-steer behavior $(a<b)$, the yaw rate limits were reached before rear slip and front tire forces limits. The yaw rate boundary varies significantly since it is inversely dependent on longitudinal velocity. We observed the constraints are satisfied and the vehicle maintained safe operation at all times. Also, a feasibility robustness margin exists even at saturating cases (Fig. 3. at $t=10 \mathrm{~s})$. It is also possible to notice that tire uncertainties cause higher amplitude disturbances on high slip angles cases rather than on small slip angles, mostly due to the significant variation in the affine term present in the dynamics (Fig. 3e and 3 at $t>7 s$ ).

\section{CONCLUSIONS}

This paper proposed a Steer-by-Wire-based lateral dynamics driver assistance system using Guaranteed Cost Model Predictive Control. Front and rear tire saturation limits where defined as operation boundaries in order to avoid lossof-control situations. Stability and feasibility robustness to tire parameter uncertainties were also incorporated to the system design to ensure safe operation on most driving conditions, since tire parameters vary with temperature, wear and manufacturing process; and cannot be assumed constant or known.

Future work on the controller will focus on the incorporation of static and dynamic objects to the controller safety envelope constraints and the mitigation of tire blowouts.

\section{REFERENCES}

[1] N. H. T. S. Administration et al., "National motor vehicle crash causation survey: Report to congress," National Highway Traffic Safety Administration Technical Report DOT HS 811 059, 2008.

[2] T. A. Badgwell and S. J. Qin, "Model-predictive control in practice," Encyclopedia of Systems and Control, pp. 756-760, 2015.

[3] C. Beal and J. Gerdes, "Model predictive control for vehicle stabilization at the limits of handling," Control Systems Technology, IEEE Transactions on, vol. 21, no. 4, pp. 1258-1269, July 2013.

[4] D. Bernardini, S. Di Cairano, A. Bemporad, and H. Tsengz, "Driveby-wire vehicle stabilization and yaw regulation: A hybrid model predictive control design," in Decision and Control, 2009 held jointly with the 2009 28th Chinese Control Conference. CDC/CCC 2009. Proceedings of the 48th IEEE Conference on. IEEE, 2009, pp. 76217626.

[5] F. Braghin, F. Cheli, S. Melzi, and F. Resta, "Tyre wear model: validation and sensitivity analysis," Meccanica, vol. 41 , no. 2 , pp. 143-156, 2006.

[6] E. Fiala, "Kraftfahrzeugtechnik," in Dubbel. Springer, 1987, pp. 941966.

[7] I. Gurobi Optimization, "Gurobi optimizer reference manual," 2015. [Online]. Available: http://www.gurobi.com

[8] H. Hamann, J. K. Hedrick, S. Rhode, and F. Gauterin, "Tire force estimation for a passenger vehicle with the unscented kalman filter," in Intelligent Vehicles Symposium Proceedings, 2014 IEEE. IEEE, 2014, pp. 814-819.

[9] P. Helnwein, C. H. Liu, G. Meschke, and H. A. Mang, "A new 3-d finite element model for cord-reinforced rubber composites-application to analysis of automobile tires," Finite elements in analysis and design, vol. 14, no. 1, pp. 1-16, 1993.

[10] Y.-H. Hsu, S. M. Laws, and J. C. Gerdes, "Estimation of tire slip angle and friction limits using steering torque," Control Systems Technology, IEEE Transactions on, vol. 18, no. 4, pp. 896-907, 2010.

[11] M. Koishi, K. Kabe, and M. Shiratori, "Tire cornering simulation using an explicit finite element analysis code," Tire Science and Technology, vol. 26, no. 2, pp. 109-119, 1998.

[12] J. Löfberg, "Yalmip: A toolbox for modeling and optimization in matlab," in Computer Aided Control Systems Design, 2004 IEEE International Symposium on. IEEE, 2004, pp. 284-289.

[13] C. M. Massera, M. H. Terra, and D. F. Wolf, "A guaranteed cost approach to robust model predictive control of uncertain linear systems," arXiv preprint arXiv:1606.03437, 2016.

[14] C. Massera Filho and D. F. Wolf, "Driver assistance controller for tire saturation avoidance up to the limits of handling," in 2015 12th Latin American Robotics Symposium and 2015 3rd Brazilian Symposium on Robotics (LARS-SBR). IEEE, 2015, pp. 181-186.

[15] W. H. Organization et al., "Youth and road safety," 2007.

[16] H. B. Pacejka and E. Bakker, "The magic formula tyre model," Vehicle system dynamics, vol. 21, no. S1, pp. 1-18, 1992.

[17] J. Rawlings, E. Meadows, and K. Muske, "Nonlinear model predictive control: A tutorial and survey," Advanced Control of Chemical Processes, pp. 203-214, 1994.

[18] E. Tönük and Y. S. Ünlüsoy, "Prediction of automobile tire cornering force characteristics by finite element modeling and analysis," Computers \& Structures, vol. 79, no. 13, pp. 1219-1232, 2001.

[19] L. Ulrich, "Top ten tech cars," Spectrum, IEEE, vol. 51, no. 4, pp. 38-47, 2014. 